February 2007

\title{
A simple explanation of the PVLAS anomaly in spontaneously broken mirror models
}

\author{
R. Foot and A. Kobakhidze ${ }^{1}$ \\ School of Physics \\ Research Centre for High Energy Physics \\ University of Melbourne, \\ Victoria 3010 Australia
}

The PVLAS anomaly can be explained if there exist millicharged particles of mass $\lesssim 0.1 \mathrm{eV}$ and electric charge $\epsilon \sim 10^{-6} e$. We point out that such particles occur naturally in spontaneously broken mirror models. We argue that this interpretation of the PVLAS anomaly is not in conflict with astrophysical constraints due to the self interactions of the millicharged particles which lead them to be trapped within stars. This conclusion also holds for a generic paraphoton model.

\footnotetext{
${ }^{1}$ E-mail: foot@physics.unimelb.edu.au, archilk@physics.unimelb.edu.au
} 
The PVLAS collaboration has obtained evidence for an anomalously large rotation of the polarization plane of light after its passage through a transverse magnetic field in vacuum[1]. One possible explanation $[2,3]$ of this result requires there to exist millicharged particles of mass

$$
m_{f} \lesssim 0.1 \mathrm{eV}
$$

and electric charge

$$
\epsilon \sim 10^{-6} e
$$

Such millicharged particles can arise naturally in gauge models with a hidden sector containing an unbroken $U(1)$ gauge factor with a kinetic mixing term with the standard $U(1)_{Y}$ gauge field $[4,5,6,7]$.

We will show in this letter that a gauge model of this general type can explain the PVLAS experiment whilst being consistent with astrophysical constraints. However, rather than work in the framework of a generic hidden sector model we consider the specific case of a spontaneously broken mirror model because such a model can also simply explain the required tiny masses for the millicharged particles.

In the mirror model the hidden sector is exactly the same as the standard sector, so that the gauge group is $G_{S M} \otimes G_{S M}\left(G_{S M} \equiv S U(3)_{c} \otimes S U(2)_{L} \otimes U(1)_{Y}\right)[5,6,7]$. For each ordinary particle there is a mirror partner, which we denote with a prime ('). An exact Lagrangian $Z_{2}$ symmetry interchanging ordinary and mirror particles is hypothesised, which means that all the couplings in the mirror sector are the same as in the ordinary sector. Note that the $Z_{2}$ symmetry can be interpreted as a parity symmetry $(x \rightarrow-x)$ if the roles of left and right chiral fermion fields are interchanged in the mirror sector.

It is known[5] that there are only 2 renormalizable and gauge invariant Lagrangian terms coupling the ordinary and mirror sector together: $U(1)_{Y}-U(1)_{Y}^{\prime}$ gauge boson kinetic mixing, and ordinary-mirror Higgs scalar interactions, i.e.

$$
\frac{\epsilon}{2} F_{\mu \nu}^{\prime} F^{\mu \nu}
$$

and

$$
\lambda \phi^{\prime \dagger} \phi^{\prime} \phi^{\dagger} \phi
$$

Most work on this model has focussed on the case where the $Z_{2}$ symmetry is unbroken by the vacuum, $\langle\phi\rangle=\left\langle\phi^{\prime}\right\rangle$, which means that the mirror particles have the same masses as their ordinary counterparts[5]. However, there is another vacuum solution to the Higgs potential where the $Z_{2}$ symmetry is spontaneously broken, with $\langle\phi\rangle=u \simeq 174 \mathrm{GeV}$ and $\left\langle\phi^{\prime}\right\rangle=0[6,7]$. This vacuum solution can be made manifest by parameterizing the most general Higgs potential in the form:

$$
V\left(\phi, \phi^{\prime}\right)=\lambda_{1}\left(\phi^{\dagger} \phi+\phi^{\prime \dagger} \phi^{\prime}-u^{2}\right)^{2}+\lambda_{2} \phi^{\prime \dagger} \phi^{\prime} \phi^{\dagger} \phi .
$$


Written in this way, $V \geq 0$ for $\lambda_{1}, \lambda_{2}>0$, and $V=0$ iff $\langle\phi\rangle=u,\left\langle\phi^{\prime}\right\rangle=0$ (or $\left.\left\langle\phi^{\prime}\right\rangle=u,\langle\phi\rangle=0\right)$.

When dynamical effects from mirror QCD condensation are included $\left\langle\phi^{\prime}\right\rangle \neq 0$. This effect arises from the Yukawa coupling terms: $h_{q} \bar{q}_{R}^{\prime} q_{L}^{\prime} \phi^{\prime}+$ H.c., which induce a linear term in $\phi^{\prime}$ in the Higgs potential [given that the mirror quarks condense: $\left\langle\bar{q}^{\prime} q^{\prime}\right\rangle=\Lambda^{\prime 3}$, with $\Lambda^{\prime} \sim 100 \mathrm{MeV}$, see Ref.[6, 7] for more details]. The net effect is to induce a small but non-zero VEV for $\left\langle\phi^{\prime}\right\rangle$ :

$$
\left\langle\phi^{\prime}\right\rangle \simeq h_{t} \Lambda^{\prime 3} / m_{\phi_{0}^{\prime}}^{2}
$$

where $h_{t} \approx 1$ is the mirror top-quark Yukawa coupling. It follows that these spontaneously broken mirror models have their mirror fermion masses suppressed by a factor $\eta \equiv\left\langle\phi^{\prime}\right\rangle /\langle\phi\rangle$, with

$$
\begin{aligned}
\eta & \simeq \frac{h_{t} \Lambda^{\prime 3}}{u m_{\phi_{0}^{\prime}}^{2}} \\
& \sim 10^{-7}\left(\frac{10 \mathrm{GeV}}{m_{\phi_{0}^{\prime}}}\right)^{2} .
\end{aligned}
$$

Thus the masses of the mirror fermions are naturally in the sub eV range - a necessary requirement to explain the PVLAS anomaly. Note that the mass of the mirror scalar is $m_{\phi_{0}^{\prime}}^{2} \simeq \lambda_{2} u^{2}$, and is experimentally weakly constrained because the scalar only couples very weakly to the ordinary particles via cubic and quartic interactions with the standard Higgs scalar $(\phi)^{2}[7]$.

It is generally argued that the astrophysical limits on epsilon are very stringent, $\epsilon \lesssim 10^{-14}$, arising from energy loss considerations in stars[9, 10]. Interestingly though, the published limits apply only in the millicharged mass region above about $1 \mathrm{eV}$ (see e.g. figure 1 of ref.[10]). For masses significantly above around $1 \mathrm{eV}$, the number density of millicharged particles in stars can become Boltzman suppressed $\left(\exp \left(-m_{e^{\prime}} / T\right)\right)$. In this situation, the mirror photon path length becomes very long and the photons can freely stream out of the hot inner core of the star - leading to the stringent limit $\epsilon \lesssim 10^{-14}$ mentioned above. It turns out that the case of light milli charged particles - with masses in the sub eV range is quite different. Because the masses are less than the temperature within the star, there is no Boltzman supression - and the mean free path of the mirror photons becomes very short. That is, the mirror particles are trapped within the stars by interactions. Let us now examine the physics in more detail.

There are basically two type of interactions: firstly, the mirror particles can interact with the ordinary particles which will produce a thermal population of $\bar{e}^{\prime}, e^{\prime}, \gamma^{\prime}$. For example, $\bar{e}^{\prime}, e^{\prime}$ pairs can be produced via virtual Bremsstrahlung photons: $p+e \rightarrow p+e+\bar{e}^{\prime}+e^{\prime}$. Secondly, there are also self interactions, such as $e^{\prime}+\bar{e}^{\prime} \leftrightarrow \gamma^{\prime}+\gamma^{\prime}, e^{\prime} \gamma^{\prime} \rightarrow e^{\prime} \gamma^{\prime}$. The cross section for the Bremsstrahlung process, and

\footnotetext{
${ }^{2}$ It is possible to decouple $\eta$ from $m_{\phi_{0}^{\prime}}$, by adding a $Z_{2}$ singlet scalar to the theory, which allows the VEV of $\phi^{\prime}$ to be made into a completely free parameter[8].
} 
elastic scattering processes such as $e^{\prime} e \leftrightarrow e^{\prime} e$ are proportional to $\epsilon^{2}$ and for $\epsilon \sim 10^{-6}$ are large enough to locally thermalize the mirror particles with the ordinary ones throughout the entire region of the star.

To see this, consider the $e^{\prime} e \rightarrow e^{\prime} e$ process as an example. If $m_{e^{\prime}} \lesssim 0.1 \mathrm{eV}$, the light mirror particles $\gamma^{\prime}, e^{\prime}, \bar{e}^{\prime}$ form a relativistic gas. The cross section for the $e^{\prime} e \rightarrow e^{\prime} e$ elastic scattering process is of order:

$$
\sigma \sim \frac{\epsilon^{2} \alpha^{2}}{s}
$$

so that the $e^{\prime}-e$ scattering length, $d$, is:

$$
\begin{aligned}
d & =\frac{1}{\sigma n_{e}} \\
& \sim \frac{(T / e V)^{2}}{\left(\epsilon / 10^{-6}\right)^{2}}\left(\frac{g / \mathrm{cm}^{3}}{\rho}\right) \text { meters }
\end{aligned}
$$

Thus, in the sun, for example, $d$ ranges from about 10 kilometers in the core to of order a meter in the outer regions. This distance is so short that the $e^{\prime}$ should be in local thermodynamic equilibrium with the ordinary matter in the sun, to a very good approximation. This means that at each point within the star a single temperature $T(r)$ may be defined. In particular, the mirror particles cannot have any net outward velocity.

Let us now consider the self interactions of the mirror particles. The cross section for the self interactions of the mirror particles are not suppressed by $\epsilon$ and can be quite large because the mirror particles $e^{\prime}, \bar{e}^{\prime}$ are so light. The cross section for the self interations of the mirror particles, for processes such as $e^{\prime}+\bar{e}^{\prime} \leftrightarrow \gamma^{\prime}+\gamma^{\prime}$, is thus relatively large, being of order:

$$
\sigma \sim \frac{\alpha^{2}}{s}
$$

leading to an interaction length of

$$
\begin{aligned}
\ell^{\prime} & =\left\langle\frac{1}{\sigma n}\right\rangle \\
& \sim \frac{1}{\alpha^{2} T} \sim \frac{10^{-4} \mathrm{~cm}}{T / k e V} .
\end{aligned}
$$

The small mean free path of the mirror particles can alleviate their impact on the transport of energy within stars - which is another important astrophysical constraint on exotic particles weakly coupled to the ordinary particles. Typically, exotic particles shouldn't transport energy faster than energy transport from ordinary photons [9].

The relevant equation governing the radiative energy transport is:

$$
L_{r}=-\frac{4 \pi r^{2}}{3 \kappa_{\gamma} \rho} \frac{d\left(a T^{4}\right)}{d r}
$$


where $L_{r}$ is the interior luminosity due to all of the energy generated within the star interior to the radius $r$. Also $a T^{4}$ is the energy density of the radiation field ( $a=\pi^{2} / 15$ in natural units) and $\kappa_{\gamma}$ is the opacity. By definition, $\left(\kappa_{\gamma} \rho\right)^{-1} \equiv \ell_{\gamma}$ is the photon mean free path. Thus, as the photon mean free path becomes smaller, energy is transported less efficiently (for a given temperature gradient). Physically this is because radiative energy transport is essentially a random walk process. If we include the contribution of the mirror particles, and write the energy transport equation in terms of the mean free paths, then we have:

$$
L_{r}=-\frac{4 \pi r^{2}}{3}\left(\ell_{\gamma}+\ell_{\gamma^{\prime}}+\ell_{e^{\prime}}+\ell_{\bar{e}^{\prime}}\right) \frac{d\left(a T^{4}\right)}{d r} .
$$

Note that $\ell_{\gamma^{\prime}}, \ell_{e^{\prime}}, \ell_{\bar{e}^{\prime}} \sim \ell^{\prime}$ (Eq.11). Thus, the mirror particles do not contribute significantly to energy transport if $\ell^{\prime} \lesssim \ell_{\gamma}$. The photon mean free path is:

$$
\ell_{\gamma}=\frac{1}{\kappa_{\gamma} \rho}
$$

with $\kappa_{\gamma}$ typically of order $\kappa_{\gamma}^{-1} \sim 1 \mathrm{~g} / \mathrm{cm}^{3}$. For the conditions within the interior of main sequence stars, $\rho \lesssim 10^{2} \mathrm{~g} / \mathrm{cm}^{3}$ and hence $\ell^{\prime} \ll \ell_{\gamma}$. Evidently, the mirror particles are typically consistent with the energy transfer constraints. We therefore conclude that this type of explanation for the PVLAS anomaly appears to be consistent with astrophysical constraints. This conclusion should also hold for a generic hidden sector model (sometimes called 'paraphoton' models in the literature) provided that the model has qualitatively similar features to the spontaneously broken mirror model. That is, has the milli electric charges of the hidden sector fermions or bosons ${ }^{3}$ induced by the kinetic mixing of the photon with a massless (or very light) hidden sector gauge boson.

Of course, even if the model is consistent with astrophysical bounds, the model will still be problematic for Big Bang Nucleosynthesis (BBN). This is because there will be a significant increase in energy density arising from the production of mirror particles, which will typically thermalize with the ordinary ones at the BBN epoch $(T \sim 1 \mathrm{MeV})$. However, the increase in energy density, could be compensated, by e.g. an electron neutrino asymmetry[11]. Therefore, the millicharged particle interpretation of the PVLAS anomaly cannot be rigorously excluded via BBN arguments. Importantly, the millicharged particle interpretation will be experimentally tested in the near future by forthcoming experiments (see. e.g. discussion in Ref.[3, 12]).

Another important issue is the impact of the model on the cosmic microwave background (CBM) measurements. The existence of the light mirror particles in local thermodynamic equilibrium with the photons might be expected to have some important effects at the recombination era because the pressure and density of the relativistic component is increased relative to the baryonic part (c.f. ref.[13]). To determine the significance of these effects a careful study might therefore be needed varying all of the known parameters (including $\Omega_{b}$ ). Post recombination, observe

\footnotetext{
${ }^{3}$ The experimental implications of millicharged bosons are quite different to fermions[3].
} 
that the photons remain in thermal equilibrium with these light mirror particles, via processes such as $\gamma^{\prime}+\gamma \leftrightarrow e^{\prime}+\bar{e}^{\prime}$. The cross section for these processes is relatively large (for $T \gtrsim m_{e^{\prime}}$ ): $\sigma \sim \epsilon^{2} \alpha^{2} / T^{2}$, leading to an interaction rate of order:

$$
\begin{aligned}
\Gamma & \sim \epsilon^{2} \alpha^{2} T \\
& \sim 0.1\left(\frac{\epsilon}{10^{-6}}\right)^{2}\left(\frac{T}{e V}\right) s^{-1}
\end{aligned}
$$

Note that this interaction rate is very many orders of magnitude larger than the explansion rate of the Universe, so that the interactions will not lead to any spectral distortions in the CMB. When $T \approx m_{e^{\prime}}$, the $e^{\prime}$ will begin to annhilate and heat the remaining $\gamma, \gamma^{\prime}$. The main effect of this heating of the CMB should simply be to increase the inferred lifetime of the Universe. Since without the heating effect, the current CMB temperature should be lower than what it is measured to be - and this would happen if the Universe were older so that a larger red shift could occur.

In conclusion, we have pointed out that the PVLAS anomaly might be explained within the context of spontaneously broken mirror models (or a generic variation, such as a generic 'paraphoton' model). These models contain a spectrum of light millicharged particles in the sub eV mass range. We have pointed out that such models are not in conflict with astrophysical constraints due to the interactions of the millicharged particles which lead them to be trapped within stars (with a mean free path short enough to evade constraints from energy transport within stars). However, the model is inconsistent with standard BBN, so if the millicharged particle interpretation of the PVLAS anomaly is experimentally confirmed, then a non-standard BBN scenario might have to be contemplated.

Acknowledgements: The authors would like to thank N. Cornish and J. Redondo for useful correspondence. This work was supported by the Australian Research Council.

\section{References}

[1] E. Zavattini et al (PVLAS Collaboration), Phys. Rev. Lett. 96, 110406 (2006).

[2] H. Gies, J. Jaeckel and A. Ringwald, Phys. Rev. Lett. 97, 140402 (2006) [ hep-ph/0607118].

[3] M. Ahlers, H. Gies, J. Jaeckel and A. Ringwald, hep-ph/0612098.

[4] B. Holdom, Phys. Lett. B166, 196 (1986), see also L. B. Okun, Sov. Phys. JETP 56, 502 (1982).

[5] R. Foot, H. Lew and R. R. Volkas, Phys. Lett. B272, 67 (1991); The concept of mirror matter was discussed prior to the advent of the standard model in $\mathrm{T}$. D. Lee and C. N. Yang, Phys. Rev. 104, 256 (1956); I. Kobzarev, L. Okun and I. Pomeranchuk, Sov. J. Nucl. Phys. 3, 837 (1966); M. Pavsic, Int. J. Theor. Phys. 9, 229 (1974). 
[6] R. Foot and H. Lew, hep-ph/9411390 (1994).

[7] R. Foot, H. Lew and R. R. Volkas, JHEP 0007, 032 (2000) [hep-ph/0006027].

[8] Z. Berezhiani and R. N. Mohapatra, Phys. Rev. D52, 6607 (1995) [hepph/9505385].

[9] G. G. Raffelt, Stars as Laboratories for Fundamental Physics, University of Chicago Press, 1996.

[10] S. Davidson, S. Hannestad and G. Raffelt, JHEP 0005, 003 (2000) [hep$\mathrm{ph} / 0001179]$.

[11] See e.g. K. A. Olive, D. N. Schramm, D. Thomas and T. P. Walker, Phys. Lett. B265, 239 (1991).

[12] S. N. Gninenko, N. V. Krasnikov and A. Rubbia, hep-ph/0612203.

[13] A. Melchiorri, A. Polosa and A. Strumia, hep-ph/0703144. 